\title{
In situ Cathodoluminescence and Monitoring Electronic Structure Change Using Optical TEM Holder
}

\author{
Mingjie Xu ${ }^{1,2}$, Sheng $\mathrm{Dai}^{1}$, Wenpei Gao ${ }^{1}$, Thomas Blum ${ }^{3}$ and Xiaoqing $\operatorname{Pan}^{1,2,3}$ \\ 1. Department of Materials Science and Engineering, University of California-Irvine, Irvine, CA, USA. \\ 2. Irvine Materials Research Institute, University of California-Irvine, Irvine, CA, USA. \\ ${ }^{3 .}$ Department of Physics and Astronomy, University of California-Irvine, Irvine, CA, USA.
}

A double tilting holder with high stability is essential for in situ transmission electron microscopy (TEM), but the availability of such holders for in situ TEM studies under various external stimuli is limited. Here, we report a unique design of seal-bearing components that provides ultra-high stability and multifunctionality (including double tilting) in an in situ TEM holder. The seal-bearing subsystem provides superior vibration damping while maintaining excellent vacuum sealing and small form factor. Combining such seal-bearing subsystem with an optical fiber assembly, CL spectroscopy as well as in situ illumination can be performed on this platform with high spatial resolution imaging inside TEM.[1] In this work, we have demonstrated the capability of this holder by several experiments including acquiring spectra on various phosphor powder, revealing electronic structure at the ZnSe/CZTSe interface, as well as photocatalytic behavior of metal/oxide interface.

ZnSe, one of the major secondary phases of CZTSe, is often found even in the highest efficient solar cell devices. However, its formation mechanism and effect on the cell performance is still controversial since classic characterization techniques are unable to provide direct correlation between nanostructures (such as precipitates) and the performance of the CZTSe solar cells. We carried out in situ off-axis holography to study the formation and electrical properties of ZnSe in CZTSe. An optical fiber passes through the holder and its rear end is coupled with a $50 \mathrm{~mW}$ laser. The laser wavelength used is $504 \mathrm{~nm}$, which is smaller than the direct bandgap of CZTSe to ensure the charge carrier properly excited. During the in situ experiment, the real-time phase mapping was recorded with the illumination on and off, and the potential mapping is then derived during the post processing. The result indicates potential well formed at the interface that attracts electrons, which affects solar cell performance by decreasing minority carrier collection.

In conclusion, we have built an in situ TEM holder that enables both high resolution TEM imaging and optical input/output. The combination of in-situ illumination and off-axis holography in TEM is proven to be a promising tool to investigate the electrical properties of nanoscale features, like $\mathrm{ZnSe}$ embedded in CZTSe. In addition, real time photocatalytic behaviors such as classical metal/oxide system can be directly observed inside TEM, which provides new perspective to the development of new generation catalyst systems [2].

References:

[1] M Xu et al., Ultramicroscopy 192 (2018), p.1.

[2] This work was supported by the Department of Energy (DOE) under Grant DE-SC0014430 and by the National Science Foundation under Grant DMR-1506535. TEM experiments were conducted in the Irvine Materials Research Institute (IMRI) at the University of California-Irvine. 


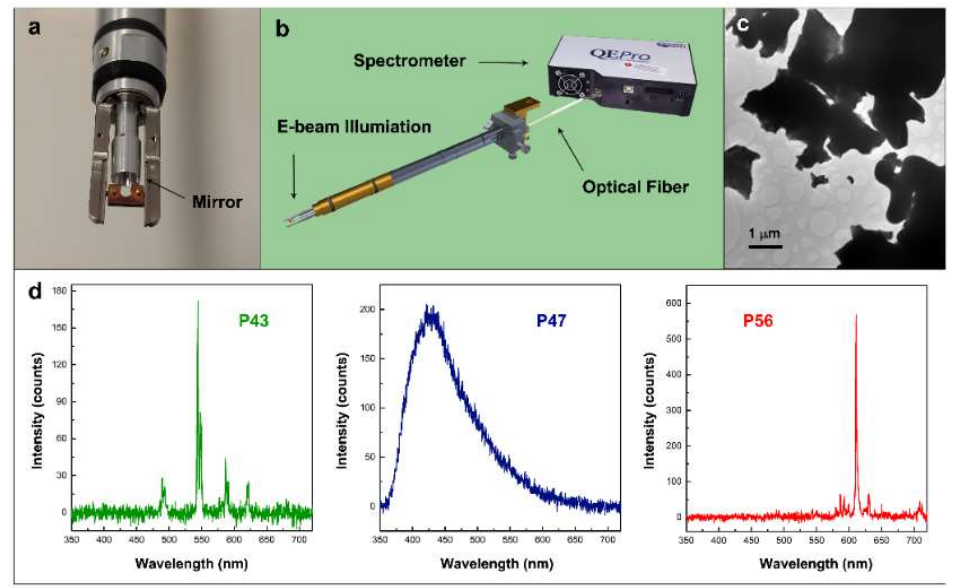

Figure 1. Experimental results of CL spectroscopy. (a) Photograph of the front end of the holder showing optical components. (b) Schematic showing the set up of in situ optical experiment. (c) TEM image of the phosphor powders dispersed on a holey carbon grid. (d) Collected optical spectra of P43 $\left(\mathrm{Gd}_{2} \mathrm{O}_{2} \mathrm{~S}: \mathrm{Tb}\right), \mathrm{P} 47\left(\mathrm{Y}_{2} \mathrm{SiO}_{5}: \mathrm{Ce}\right)$, and $\mathrm{P} 56\left(\mathrm{Y}_{2} \mathrm{O}_{3}: \mathrm{Eu}\right)$ phosphor powder samples.

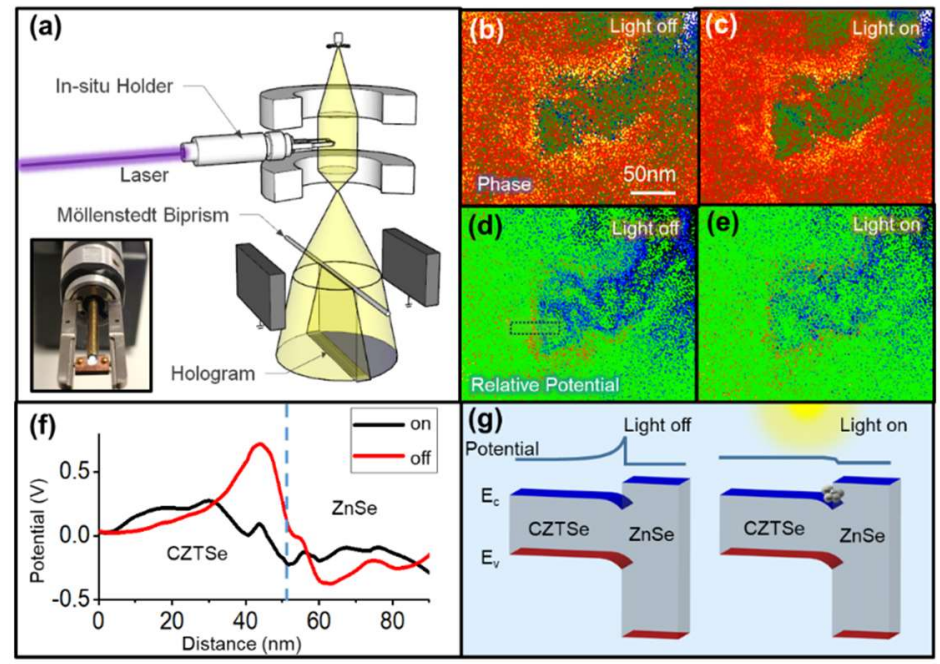

Figure 2. In-situ holography mapping with optical illumination on a ZnSe imbedded in CZTSe. (a) is a schematic of in-situ illuminated off-axis holography setup in TEM with imbedded photo of the optical TEM holder(b), (c), (d) and (e) are the phase mapping and potential mapping around a ZnSe precipitate imbedded in a CZTSe matrix with illumination off and on. (f) is the potential line profile across the interface marked in (d). The CZTSe matrix is used as a zero-potential reference. (g) is the schematic of band diagram and corresponding potential distribution in different illumination condition. 